OPEN ACCESS

Edited by: Lorenzo Manti,

University of Naples Federico II, Italy

Reviewed by:

Pavel Blaha,

National Institute of Nuclear Physics of

Naples, Italy

Martina C. Fuss,

GSI Helmholtz Center for Heavy Ion

Research, Germany

${ }^{*}$ Correspondence:

Gabriel Adrian

gabriel.adrian@med.lu.se

Specialty section:

This article was submitted to

Radiation Oncology,

a section of the journal

Frontiers in Oncology

Received: 26 March 2021

Accepted: 07 July 2021

Published: 29 July 2021

Citation:

Adrian G, Konradsson E, Beyer S,

Wittrup A, Butterworth KT,

McMahon SJ, Ghita M,

Petersson K and Ceberg C (2021)

Cancer Cells Can Exhibit a Sparing

FLASH Effect at Low Doses Under

Normoxic In Vitro-Conditions.

Front. Oncol. 11:686142.

doi: 10.3389/fonc.2021.686142

\section{Cancer Cells Can Exhibit a Sparing FLASH Effect at Low Doses Under Normoxic In Vitro-Conditions}

\author{
Gabriel Adrian ${ }^{1,2 *}$, Elise Konradsson ${ }^{3}$, Sarah Beyer ${ }^{1}$, Anders Wittrup ${ }^{1,2,4}$, \\ Karl T. Butterworth ${ }^{5}$, Stephen J. McMahon ${ }^{5}$, Mihaela Ghita ${ }^{5}$, Kristoffer Petersson ${ }^{2,6}$ \\ and Crister Ceberg ${ }^{3}$ \\ ${ }^{1}$ Division of Oncology and Pathology, Clinical Sciences, Skåne University Hospital, Lund University, Lund, Sweden, \\ 2 Department of Hematology, Oncology and Radiation Physics, Skåne University Hospital, Lund, Sweden, ${ }^{3}$ Department of \\ Medical Radiation Physics, Clinical Sciences, Lund University, Lund, Sweden, 4 Wallenberg Center for Molecular Medicine, \\ Lund, Sweden, ${ }^{5}$ Patrick G. Johnston Centre for Cancer Research, Queen's University Belfast, Belfast, United Kingdom, \\ ${ }^{6}$ Medical Research Council Oxford Institute for Radiation Oncology, Department of Oncology, University of Oxford, Oxford, \\ United Kingdom
}

Background: Irradiation with ultra-high dose rate (FLASH) has been shown to spare normal tissue without hampering tumor control in several in vivo studies. Few cell lines have been investigated in vitro, and previous results are inconsistent. Assuming that oxygen depletion accounts for the FLASH sparing effect, no sparing should appear for cells irradiated with low doses in normoxia.

Methods: Seven cancer cell lines (MDA-MB-231, MCF7, WiDr, LU-HNSCC4, HeLa [early passage and subclone]) and normal lung fibroblasts (MRC-5) were irradiated with doses ranging from 0 to 12 Gy using FLASH ( $\geq 800$ Gy/s) or conventional dose rates (CONV, 14 $\mathrm{Gy} / \mathrm{min}$ ), with a $10 \mathrm{MeV}$ electron beam from a clinical linear accelerator. Surviving fraction (SF) was determined with clonogenic assays. Three cell lines were further studied for radiation-induced DNA-damage foci using a 53BP1-marker and for cell cycle synchronization after irradiation.

Results: A tendency of increased survival following FLASH compared with CONV was suggested for all cell lines, with significant differences for $4 / 7$ cell lines. The magnitude of the FLASH-sparing expressed as a dose-modifying factor at $\mathrm{SF}=0.1$ was around 1.1 for $6 /$

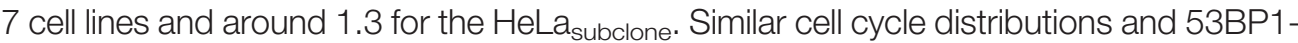
foci numbers were found comparing FLASH to CONV.

Conclusion: We have found a FLASH effect appearing at low doses under normoxic conditions for several cell lines in vitro. The magnitude of the FLASH effect differed between the cell lines, suggesting inherited biological susceptibilities for FLASH irradiation.

Keywords: FLASH, ultra-high dose rate irradiation, clonogenic assay, normoxia, radiotherapy, radiobiology, radioresistance, cancer cell lines 


\section{INTRODUCTION}

The FLASH effect denotes the radiobiological phenomenon that a given absorbed dose of ionizing radiation produces less damage at ultra-high dose rates $(>40-100 \mathrm{~Gy} / \mathrm{s})$, as compared to the lower dose rates conventionally used in radiotherapy (CONV, about $0.1 \mathrm{~Gy} / \mathrm{s}$ ). Experimental evidence for the FLASH effect has been demonstrated in vivo in various preclinical settings (1-5), as well as in one first-in-human case of a patient with multi-resistant cutaneous lymphoma (6).

Although there is no proven mechanistic explanation for the FLASH effect, the limited in vivo data available suggest that more sparing may occur in physoxic normal tissues than in severely hypoxic or nearly anoxic tumors (7). One plausible theory describes the FLASH effect as a protective, radiation-induced hypoxia, tentatively explained by the so-called transient oxygen depletion (TOD) hypothesis as a net effect of radiolytic oxygen consumption exceeding the physiologic supply (8-10). According to the TOD hypothesis, the degree of sparing would be largest for already hypoxic tissues, where further oxygen depletion can be substantial. No effect would be expected at normoxia, where radiolytic oxygen consumption would not be sufficient for producing hypoxic radioresistance, or at anoxia where there can be no further oxygen depletion. However, recent work by Labarbe et al. has indicated, based on simulations and mathematical modelling, that the TOD hypothesis is most likely not sufficient to account for the FLASH effect reported at dose levels limited by normal tissue toxicity (11). Consequently, the authors suggest that other mechanisms may regulate the process and that a FLASH effect may be present also at normoxic conditions, even at relatively low dose levels.

Surprisingly, few recent in vitro studies with clonogenic assays have been reported to support these basic assumptions, and the limited data available suggests that different cell lines may have different susceptibility to the FLASH effect. In previous work, we studied clonogenic survival of the human prostate cancer DU145 cell line and found a FLASH effect at lower oxygen concentrations but no significant differences in normoxic conditions (12). Montay-Gruel et al. studied the murine glioblastoma $\mathrm{H} 454$ cell line and demonstrated significant FLASH effects both at $4 \%$ oxygen concentration and in normoxic conditions (13). For normal human lung fibroblasts (14) and lung cancer A549 (15), no difference in survival at different dose-rates in normoxia was reported. Neither has the use of laser-accelerated protons revealed any dose-rate dependent differences in normoxia (16-18). Contrary to these findings, for two murine pancreatic cancer cell lines, Venkatesulu et al. found a reversed FLASH effect at normoxic conditions (19).

Consequently, there is a need for further in vitro studies allowing for experiments in a controlled oxygen environment $(1,20)$. In the present work, we have performed a comparative study of FLASH vs. CONV and assessed clonogenic survival, DNA damage, and cell cycle synchronization under normoxic conditions for a range of different cell lines. Our investigations show that the FLASH effect may occur at relatively low doses under normoxic conditions and that it depends on cell-line specific variations in susceptibility.

\section{MATERIAL AND METHODS}

\section{Cell Culture}

The human breast cancer cell lines MCF7 and MDA-MB-231, the human fibroblast cell line MRC-5, and the human cervix cancer cell line HeLa (in the study two different HeLa cells were used; early passage cells and a high passage subclone) were acquired from American Type Culture Collection (ATCC). The human colon cancer cell line $\mathrm{WiDr}$ was acquired from LGC Promochem (Teddington, UK). The squamous cell carcinoma LU-HNSCC4 was established in our laboratory from a patient with a squamous cell carcinoma in the floor of the mouth (21). Cells were grown in monolayers in DMEM (MCF7, MDA-MB-231, WiDr, HeLa, LU-HNSCC4) or EMEM (MRC5) media with $10 \%$ fetal bovine serum and $1 \%$ penicillinstreptomycin at $37^{\circ} \mathrm{C}$ in a humidified atmosphere with $5 \%$ $\mathrm{CO}_{2}$. All cell lines were confirmed to be negative for mycoplasma infection.

\section{Clonogenic Assays}

Exponentially growing cells were trypsinized and plated in appropriate cell densities in $2.50 \mathrm{ml}$ medium per Falcon T12.5 flask (Thermo Fischer Scientific TM, Waltham, MA) and allowed to adhere overnight before irradiation. Control flasks for determination of the plating efficiency and the FLASH- and CONV-flasks were prepared identically on the same occasion, for each repetition. FLASH and CONV-flasks were irradiated minutes apart with doses from 0-9 Gy (12 Gy for HeLa $\mathrm{L}_{\text {subclone }}$ ). Irradiation was performed under normoxic conditions at room temperature with the flasks lying flat and irradiated from beneath (beam angle 180 degrees). After irradiation, the flasks were returned to the incubator for 9-14 days. All flasks, including the non-irradiated controls, were terminated at the same occasion. Cells were fixed and stained with methylene-blue in $70 \%$ ethanol. Flasks were scanned using a flatbed scanner in 1,200 dpi resolution. Colony counts were performed with a standardized ImageJ-code (version 1.53e, Wayne Rasband, National Institute of Health, USA) and manually checked. Surviving fraction (SF) was determined as the number of colonies with at least 50 cells divided by the number of plated cells (corrected for plating efficiency).

\section{DNA-Double Strand Break Foci Formation}

150000 - 500000 cells were plated in Slide-Flasks (Thermo Fischer Scientific Nunc, Roskilde, Denmark) and allowed to adhere overnight before irradiation with $3 \mathrm{~Gy}$. At specific time points after irradiation, cells were washed with PBS and fixed with $4 \%$ paraformaldehyde for $20 \mathrm{~min}$. After washing, cells were permeabilized with $0.5 \%$ Triton-X100 in PBS for $20 \mathrm{~min}$, washed, blocked in blocking buffer $(0.2 \%$ skimmed milk, $0.1 \%$ TritonX$100,5 \%$ FBS in PBS) for 1 hour followed by 1 hour incubation with 53BP1 primary antibody (Invitrogen PA146147) and 1 hour of incubation with a secondary antibody (AlexaFluor anti-rabbit 488). Cell nuclei were counterstained with DAPI. Permeabilization, washing, blocking and staining steps were all performed at room temperature. Foci formation were assessed with a widefield fluorescence microscope, AxioOberver Z.1 
(Zeiss, Oberkochen, Germany), equipped with ×63/1.40 PlanApochromat oil-immersion objective lens and Colibri 7 solid state LED light source (Zeiss), and an ORCA-Flash4.0 V3 Digital CMOS camera (Hamamatsu Photonics, Hamamatsu City, Japan). In each sample, at four different positions, fifteen Zstack images were acquired, deconvoluted with a GPU-based deconvolution module and averaged using a Maximum Intensity Projection-algorithm (Black Zen Imaging Software, Zeiss). ImageJ was used for automated foci identification and quantification of DNA-double strand break (DSB) foci.

\section{Cell Cycle Analyses}

150000 - 500000 cells were plated in $35 \mathrm{~mm}$ or $60 \mathrm{~mm}$ Petri Dishes (Corning, Corning, NY, USA) and allowed to adhere overnight before irradiation with $6 \mathrm{~Gy}$ (and $3 \mathrm{~Gy}$ for the

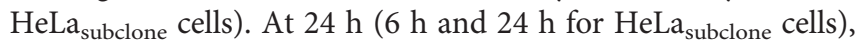
after irradiation cells were washed with PBS, harvested and fixated with ice-cold ethanol (70\%). Cell nuclei were stained with propidium iodide $(10 \mathrm{lg} / \mathrm{ml}$, RNase A $0.1 \mathrm{lg} / \mathrm{ml})$ for $30 \mathrm{~min}$ at room temperature and DNA content was determined with an Accuri C6 Flow Cytometer (Becton Dickinson, Franklin Lakes, NJ, USA). DNA-histograms were analyzed in ModFit LT 5.0 for Mac (BD Biosciences).

\section{Irradiation and Dosimetry}

Irradiation and dosimetry were performed as described previously (12). In summary, a modified (22) Elekta Precise (Elekta AB, Stockholm, Sweden) medical linear accelerator (LINAC) was used for irradiations with FLASH and CONV dose rates with a $10 \mathrm{MeV}$ electron beam. The average dose rate for CONV irradiation was $14 \mathrm{~Gy} / \mathrm{min}$. For FLASH irradiation, the average dose rate was $\geq 800 \mathrm{~Gy} / \mathrm{s}$, delivered with an integer number of $3.5 \mu$ s pulses, with a dose-per-pulse of $3.0 \mathrm{~Gy}$, and a pulse repetition frequency of $200 \mathrm{~Hz}$. Thus, the instantaneous pulse dose rate was $0.86 \mathrm{MGy} / \mathrm{s}$, which is the same as the average dose rate for the 3 Gy single pulse delivery, while the average dose rate was $1.2 \mathrm{kGy} / \mathrm{s}$ for the $6 \mathrm{~Gy}$ delivery, $900 \mathrm{~Gy} / \mathrm{s}$ for the 9 Gy delivery, and $800 \mathrm{~Gy} / \mathrm{s}$ for the 12 Gy delivery. GafChromic EBT3 film (Ashland Specialty Ingredients G.P., Bridgewater, NJ) was used for dosimetry for both FLASH and CONV irradiation. Dose measurements were performed in conjunction with each cell experiment. In addition, online dose delivery verification measurements were performed. For CONV irradiation, these were performed with the built-in monitor (transmission) chamber. For FLASH irradiation, a Farmer-type ionization chamber placed at a specific position in the ceiling of the treatment room (furthest possible distance from the source) was used.

\section{Statistical Analyses}

RStudio v. 1.0.136 (RStudio Team (2015). RStudio, Inc., Boston, MA, URL http://www.rstudio.com/) was used for statistical calculations. The parameters of the linear-quadratic model (23) $\left(\mathrm{SF}=\exp \left(-\alpha \mathrm{D}-\beta \mathrm{D}^{2}\right)\right)$ were fitted to the $\log (\mathrm{SF})$ using the nonlinear least-squares method ('nls' in RStudio). Two alternative models were fitted, one with separate $\alpha$ and $\beta$ parameters for the CONV and FLASH data, and one with common $\alpha$ and $\beta$ parameters for all data. The residuals were tested for normality using the Kolmogorov-Smirnov test, and the F-test was used to determine whether the fit was significantly improved by using separate parameters. Using the model fitted with separate $\alpha$ and $\beta$ parameters for the CONV and FLASH data, dose-modifying factors (DMF) were determined as the ratio of $\mathrm{D}_{\text {FLASH }} / \mathrm{D}_{\mathrm{CONV}}$ at a survival fraction of $0.1(\mathrm{SF}=0.1)$ and 0.01 $(\mathrm{SF}=0.01)$. Boot-strapping was used to calculate the median and the interquartile range of the DMF. In addition, the difference in survival fraction at the individual dose levels were tested for statistical significance, without assuming normality, by using the Wilcoxon rank-sum test. All tests were two-sided with a chosen significance level of 5\%. Experiments were repeated 2-4 times.

\section{RESULTS}

\section{Clonogenic Assays}

A tendency of increased survival after FLASH compared with CONV was suggested for all cell lines (Figure 1), with significant differences for four of the seven cell lines. A general FLASHsparing was also indicated by a DMF at $\mathrm{SF}=0.1$ of around 1.1 for all cell lines, except the $\mathrm{HeLa}_{\text {subclone, }}$ for which it was around 1.3 (Table 1). The use of separate $\alpha$ and $\beta$ parameters for FLASH and CONV resulted in significantly improved fits for the MCF7, LU-HNSCC4, HeLa $a_{\text {early passage }}$ and $\mathrm{HeLa}_{\text {subclone, indicating }}$ differences across the curves as a whole. Significant survival differences were also observed for MDA-MB-231 at 6 Gy and 9 Gy. The WiDr and MRC-5 cell lines did not show any significant differences in survival after FLASH compared with CONV in the dose range studied.

\section{DSB-Foci With 53BP1}

Three of the cancer cell lines, LU-HNSCC4, MDA-MB-231 and $\mathrm{HeLa}_{\text {subclone, }}$ were further studied for radiation-induced DNADSB using the 53BP1-marker at $2 \mathrm{~h}$ and $24 \mathrm{~h}$ after irradiation with 3 Gy (Figure 2). A marked induction of DNA-DSB foci was seen at $2 \mathrm{~h}$ after irradiation, and declined substantially at $24 \mathrm{~h}$. Comparing FLASH and CONV, median foci numbers were similar with overlapping interquartile ranges, for all three studied cell lines (Table 2 ).

\section{Cell Cycle Analyses}

To further investigate potential differences in radiation response between FLASH and CONV, radiation-induced cell cycle arrest was investigated for MDA-MD-231, LU-HNSCC4 and HeLa subclone cells. At 24 hours after irradiation with $6 \mathrm{~Gy}$, both FLASH and CONV induced cell cycle synchronizations in the three cell lines (Figures 3A-C). Interestingly, $\mathrm{HeLa}_{\text {subclone }}$ cells were predominantly synchronized in early S-phase (the S-phase was sub-analyzed in three compartments, Supplementary Figure S1), whereas the MDA-MB-231 and LU-HNSCC4 cells were synchronized in the

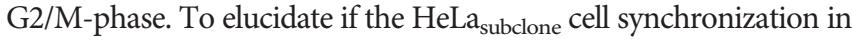
early S-phase was due to a previous transient G2/M-arrest, we studied an earlier time point, $6 \mathrm{~h}$ after irradiation and an additional dose level, $3 \mathrm{~Gy}$, and found radiation-induced G2/M-arrest 

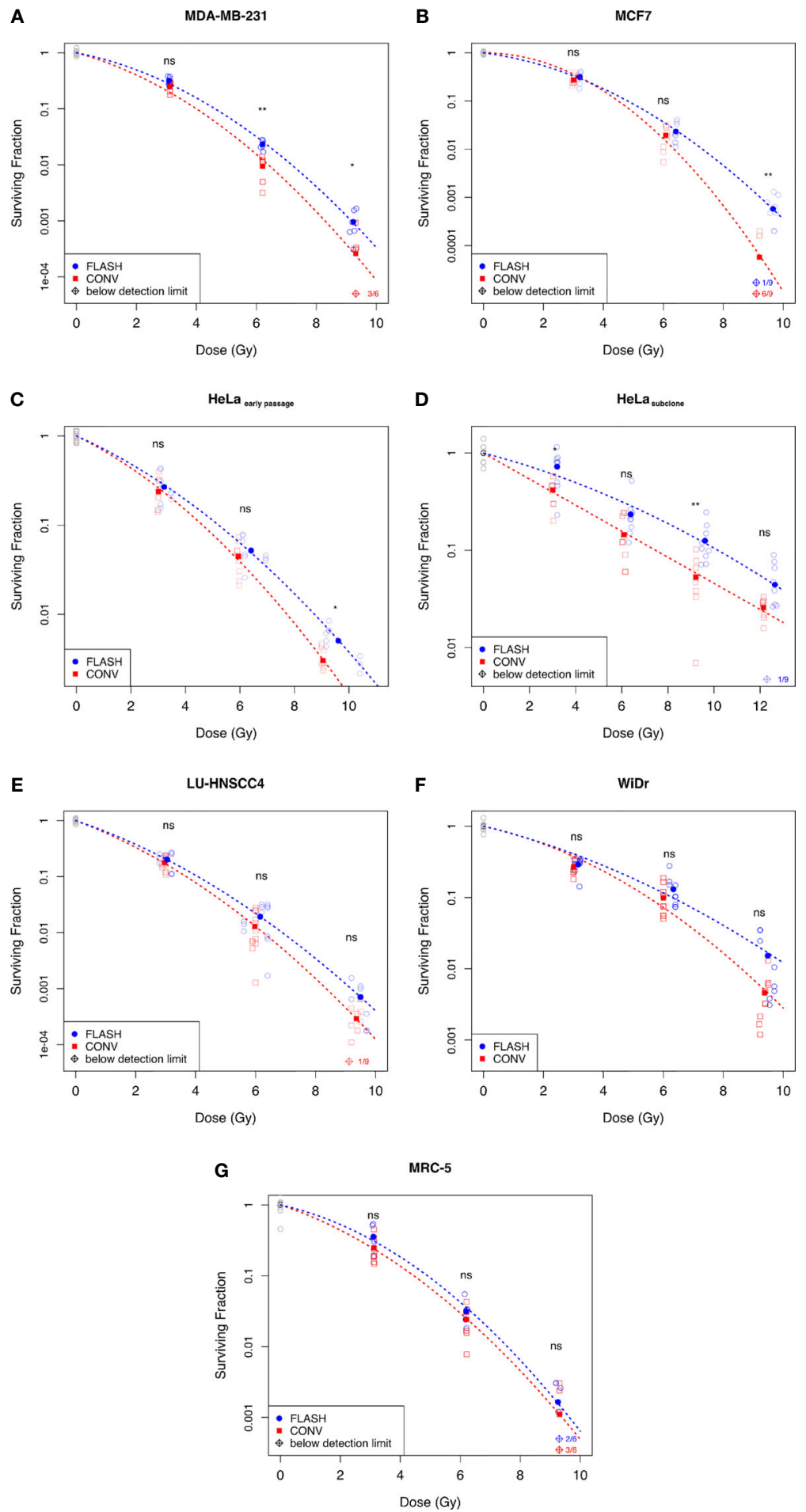

FIGURE 1 | Surviving fraction assessed by clonogenic assay comparing FLASH with conventional dose rates (CONV) for human in vitro-cell lines; (A) Breast cancer cell line MDA-MB-231, (B) Breast cancer cell line MCF7, (C) Cervix cancer cell line HeLa early passage, (D) HeLa $a_{\text {subclone }}$ (E) Head\&neck cancer cell line LU-HNSCC4, (F) Colon cancer cell line WiDr, and (G) Normal lung fibroblasts MRC-5. Blue circles denote FLASH, red squares denote CONV, and grey circles denote the nonirradiated controls. The empty symbols represent the individual flasks and the filled symbols represent the average surviving fraction at the dose indicated. The dashed lines illustrate the fitted survival curve according to the linear quadratic model. Diamond symbols denote samples below the detection limit (no surviving colonies). Statistical analyses using Wilcoxon Rank-Sum test; ns, not significant, ${ }^{\star} p<0.05,{ }^{* *} p<0.01$. Data from three independent experiments. 
TABLE 1 | Dose modifying factors (DMF) at a surviving fraction (SF) of 0.1 and 0.01 for the various cell lines.

\begin{tabular}{|c|c|c|c|c|c|}
\hline Cell line & DMF (SF=0.1) & IQR (SF=0.1) & DMF (SF=0.01) & IQR (SF=0.01) & F-test $p$-value \\
\hline WiDr & 1.16 & $1.03-1.29$ & 1.20 & $1.10-1.30$ & 0.34 \\
\hline MCF7 & 1.10 & $1.00-1.20$ & 1.16 & $1.11-1.21$ & 0.03 \\
\hline LU-HNSCC4 & 1.12 & $0.94-1.30$ & 1.15 & $1.06-1.24$ & 0.007 \\
\hline MRC-5 & 1.09 & $0.97-1.21$ & 1.05 & $1.00-1.10$ & 0.24 \\
\hline MDA-MB-231 & 1.14 & $1.02-1.26$ & 1.12 & $1.07-1.17$ & 0.15 \\
\hline HeLa ${ }_{\text {early passage }}$ & 1.12 & $1.02-1.22$ & 1.13 & $1.09-1.17$ & 0.04 \\
\hline $\mathrm{HeLa}_{\text {subclone }}$ & 1.32 & $1.19-1.45$ & NA & NA & 0.05 \\
\hline
\end{tabular}

The F-test denotes the significance level for separate parameter sets for FLASH and CONV, compared with one common fit. IQR; inter-quartile range; NA, Not Applicable.

(Figure 3D). The cell cycle analyses could not resolve any significant differences between FLASH and CONV.

\section{DISCUSSION}

We have found in vitro evidence of a FLASH sparing effect measured with clonogenic survival occurring under normoxic conditions for several cancer cell lines. The magnitude of the FLASH effect differed between the cell lines and was most pronounced for HeLa $\mathrm{Hubclone}_{\text {cells, }}$ with a significant sparing already apparent at $3 \mathrm{~Gy}$. The normal lung fibroblasts did not show any significant difference in survival between FLASH and CONV. Cell cycle synchronization and DSB-foci formation were assessed for three of the cancer cell lines with similar responses for FLASH and CONV exposures.

It is well recognized that FLASH spares normal tissues in vivo $(2-4,13,24)$. However, available in vitro results with clonogenic assays in normoxia are inconsistent both in recent $(12-15,19)$ and older studies (25-30). Consistent with our current findings, an increased survival fraction after irradiation with ultra-high

A
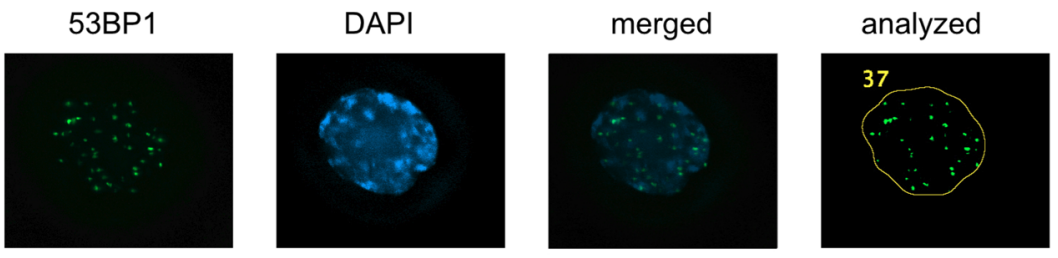

B LU-HNSCC4

C MDA-MB-231

D HeLa
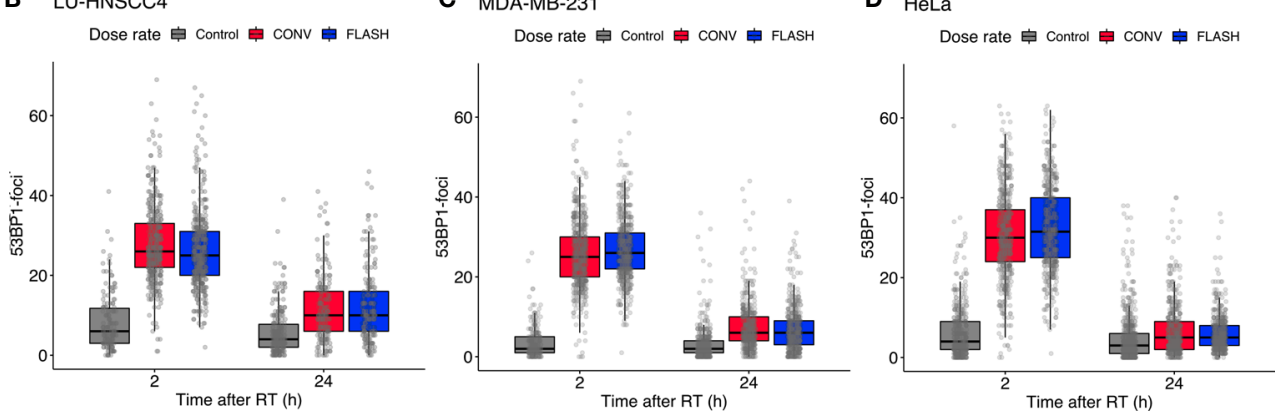

FIGURE 2 | Evaluation of radiation-induced DNA-double strand break foci using 53BP1. (A) Representative microscopy image showing (left to right) 53BP1, DAPI, merged image, and the resulting analyzed image after processing in ImageJ. (B-D) Number of 53BP1 foci at $2 \mathrm{~h}$ and $24 \mathrm{~h}$ after 3 Gy irradiation with FLASH (blue) or conventional dose rate (CONV, red) compared with controls (grey) for LU-HNSCC4 (B; 1,532 scored cells), MDA-MB-231 (C; 2,583 scored cells), and HeLasubclone (D; 2,973 scored cells). The box and whisker plots illustrate median (thick line), interquartile range (box) and the lowest/highest observation within $\pm 1.5^{\star}$ interquartile range (IQR) from the box (whiskers). The individually scored cells are indicated with transparent circles. Data from two independent experiments.

TABLE 2 | Number of DSB-foci per cell with the 53BP1-marker for three cell lines at $2 \mathrm{~h}$ and $24 \mathrm{~h}$ after irradiation with FLASH or conventional dose rate (CONV), and for non-irradiated controls (Ctrl).

\begin{tabular}{|c|c|c|c|c|c|c|}
\hline Cell line & $\begin{array}{c}\text { Ctrl at } 2 \mathrm{~h} \\
\text { Median (IQR) }\end{array}$ & $\begin{array}{l}\text { FLASH at } 2 \mathrm{~h} \\
\text { Median (IQR) }\end{array}$ & $\begin{array}{l}\text { CONV at } 2 \mathrm{~h} \\
\text { Median (IQR) }\end{array}$ & $\begin{array}{c}\text { Ctrl at } 24 \mathrm{~h} \\
\text { Median (IQR) }\end{array}$ & $\begin{array}{l}\text { FLASH at } 24 \mathrm{~h} \\
\text { Median (IQR) }\end{array}$ & $\begin{array}{l}\text { CONV at } 24 \mathrm{~h} \\
\text { Median (IQR) }\end{array}$ \\
\hline LU-HNSCC4 & $6(3-12)$ & $25(20-31)$ & 26 (22-33) & $4(2-8)$ & $10(6-16)$ & $10(6-16)$ \\
\hline HeLa subclone $_{\text {e }}$ & $4(2-9)$ & $32(25-40)$ & $30(24-37)$ & $3(1-6)$ & $5(3-8)$ & $5(2-9)$ \\
\hline
\end{tabular}

$I Q R$, Inter-quartile range. 
A

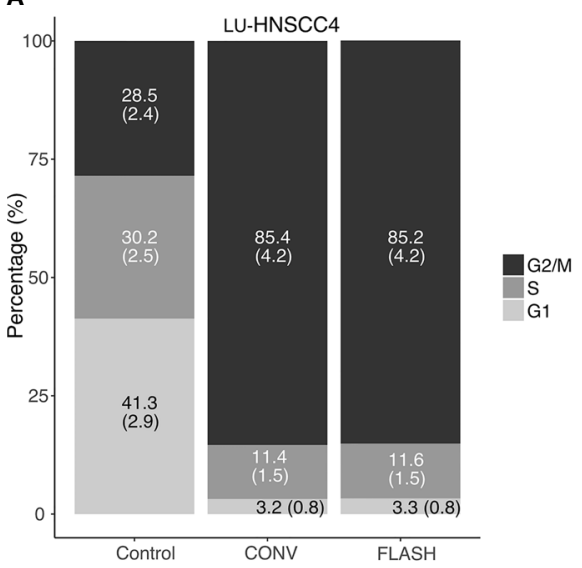

c

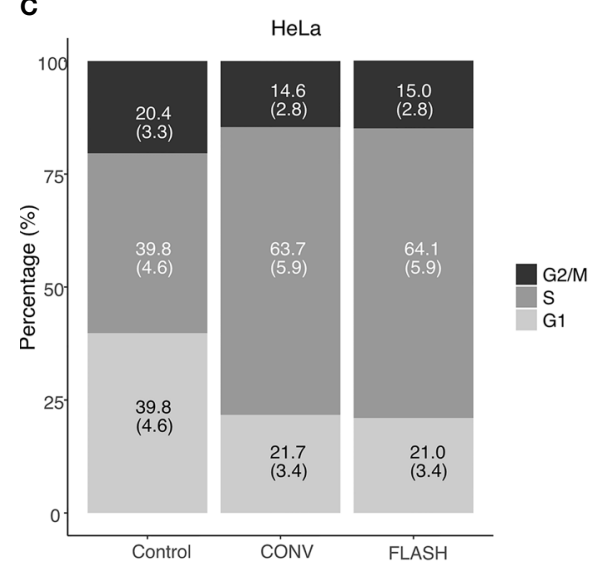

B

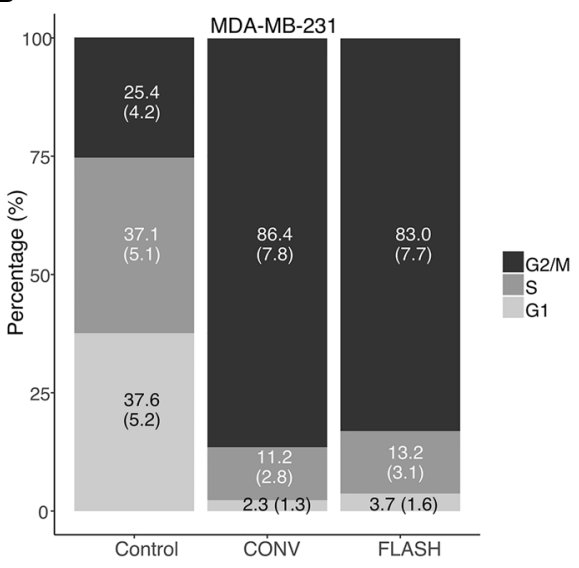

D

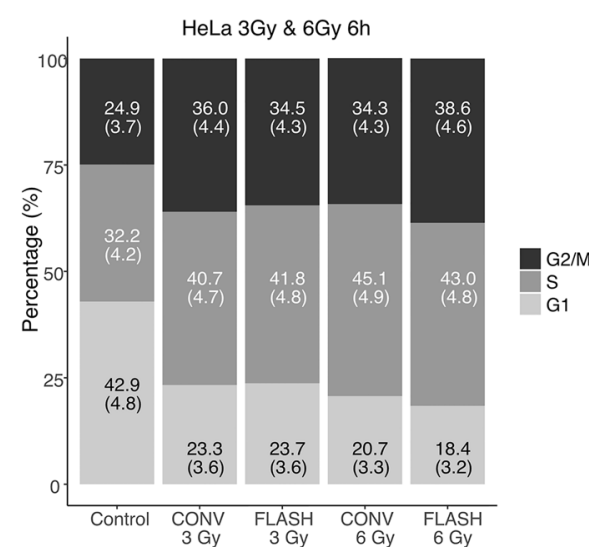

FIGURE 3 | Cell cycle distributions determined by flow cytometry after irradiation with FLASH or conventional dose rate (CONV). In (A-C), cell cycle distribution $24 \mathrm{~h}$ after irradiation with 6 Gy for LU-HNSCC4 (A), MDA-MB-231 (B) and HeLasubclone (C). In (D) an earlier time-point (6 h) after irradiation with 3 Gy and 6 Gy using the HeLa $a_{\text {subclone. }}$ Bars illustrate G1 (light grey), S-phase (grey), and G2/M (black). The figures in the bars denote the percentage of cells (with standard deviations). Data from two independent experiments.

dose rate in normoxia have been reported $(13,29,30)$, whereas other results indicate no difference $(14,15,25-28)$ or a reversed effect (19). The inconsistent results could indicate differing intrinsic biological susceptibility for FLASH. In addition, survival differences have been shown for hypoxic in vitroconditions $(12,27,28,31,32)$. In the present study, the magnitude of the FLASH effect, expressed as DMF at $\mathrm{SF}=0.1$, was around 1.1 for six of the seven cell lines, while a DMF of 1.3

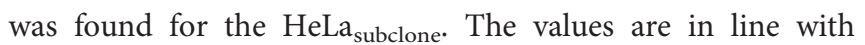
previously published in vivo data, generally showing a DMF of 1.2-1.5 (7). The HeLa $\mathrm{Hubclone}_{\text {data }}$ (with passage number approaching 40) show a distinct behavior compared to the other cell lines, with a larger DMF and also earlier cell cycle arrest. In comparison with $\mathrm{HeLa}$ early passage, the $\mathrm{HeLa}_{\text {subclone }}$ was considerably more radioresistant and lacked a shouldered survival curve for the CONV-irradiated samples. It has previously been reported that phenotype changes can occur with high passage numbers, affecting radiation responses (33).
The present results showing differences between cell lines, together with the inconsistent findings by others, suggest that the FLASH effect might not be an independent, universal dosemodifying factor. Instead, the sparing effect could involve biological determinants varying from cell line to cell line.

DSB-foci formations were numerically in the same range for the three studied cancer cell lines, with similar foci numbers for FLASH compared with CONV irradiation. Fouillade et al., using the same DSB-marker at an earlier time point (30 minutes), showed a lower number of foci for FLASH compared with CONV for normal lung fibroblasts, but no differences for the A549 lung cancer cell line (24). Cell cycle synchronization after irradiation was seen for the three studied cell lines in the current study, but with similar effects after both FLASH and CONV irradiation. Auer et al. also studied cell cycle synchronization of HeLa cells after irradiation with 3 Gy using laser-accelerated protons at different dose rates. They found a less pronounced $\mathrm{G} 2 / \mathrm{M}$-accumulation at $10 \mathrm{~h}$ for cells irradiated with ultra-high 
dose rate compared with conventional dose rate, but no differences at $24 \mathrm{~h}$ (34). The $\mathrm{HeLa}_{\text {subclone }}$ cells used in the current study revealed no synchronization in G2/M-phase at $24 \mathrm{~h}$ after irradiation, instead the cells were synchronized in early $\mathrm{S}$-phase. Additional experiments at an earlier time-point $(6 \mathrm{~h}$ after irradiation) with two different doses (3 Gy and 6 Gy) indicated an earlier radiation induced G2/M-synchronization, suggesting a peak of the G2/M-arrest at a time point before $24 \mathrm{~h}$ (Supplementary Figure S1). However, the cell cycle synchronization was similar after FLASH and CONV at both $6 \mathrm{~h}$ and $24 \mathrm{~h}$.

FLASH effects are typically seen at doses $\geq 10 \mathrm{~Gy}$. The current study showed a separation of the survival curves at doses below 10 Gy. Interestingly, also using a low dose of 4 Gy, Chabi et al. found FLASH irradiation to be more efficient than conventional dose rate exposures for two cases of T-cell lymphoblastic leukemia (T-ALL), but an opposite relation for a third case (35). The results underpin that FLASH effects do not exclusively occur at high doses and also suggest that intrinsic biological factors might determine the FLASH response. The authors proposed that genomic profiles might predict when FLASH is beneficial. Additional investigations in the nature and mechanism of such biological determinants, and their influence on the radiochemical and biological steps of the radiation response remain to be investigated. Many of the steps are likely to be influenced by the available oxygen concentration, and we have previously shown the dependence on oxygen concentration for a FLASH effect (12). However, differences between FLASH and CONV at low doses in well-oxygenated environments, i.e. where oxygen depletion is considered to be negligible, imply that the TOD hypothesis is insufficient to account for the whole FLASH effect. We therefore deduce that the FLASH effect, in part, must be caused by other mechanisms.

FLASH radiotherapy is a promising new technique and convincing reports show its ability to protect normal tissue from radiation damage $(2-5,13,24)$. Most in vivo experiments also suggest an iso-effective tumor control compared with CONV (2, 5, 36-38), even though some studies have found other results $(19,35)$. The tumor's response to radiation in the complex in vivo environment is dependent not only on direct cell kill but also on inflammatory reactions and the immune system, involving surrounding connective tissues. Considering a possible inherited susceptibility for cancer cells to exhibit a FLASH effect (35), which would be detrimental for tumor control, further investigation in the differential response between tumor and normal tissue is clearly indicated. Ideally, such studies will generate models that describe tissues and tumors for which a differential response can be exploited.

The current study has some limitations. Since the FLASH irradiation was delivered with an integer number of pulses (1-4), the average dose rate varied between the different dose levels. However, the dose-per-pulse and the instantaneous/pulse dose rate were constant and the average dose rate exceeded $800 \mathrm{~Gy} / \mathrm{s}$ at all dose levels. Further, we have chosen to perform a pure in vitro study to enable studies of different cell lines under wellcontrolled oxygen concentrations where the impact of biological interactions was minimized. Thereby, the results are valid under these circumstances, and their generalizability to more complex biological systems need to be further investigated. The lack of a difference in the DSB-foci induction between FLASH and CONV warrants future experiments using additional methods to assess DNA-damage and repair after irradiation. The varying results between cell lines suggest that biological determinants may affect the response, but this study alone does not identify any underlying mechanisms or predictive signatures that could be further examined in a preclinical or clinical translation.

To conclude, we have found a FLASH effect under normoxic conditions for several cell lines in vitro, and that the magnitude of the FLASH effect differed between the cell lines. The results indicate that the FLASH effect cannot be solely explained by TOD and that other mechanisms are involved. The nature of such possible biological susceptibilities and their dependence on oxygen concentrations will be subject to further investigations.

\section{DATA AVAILABILITY STATEMENT}

The datasets presented in this article are not readily available but the datasets generated during and/or analyzed during the current study are available from the corresponding author on reasonable request. Requests to access the datasets should be directed to gabriel.adrian@med.lu.se.

\section{AUTHOR CONTRIBUTIONS}

GA, CC, SM, and KP designed the study. GA, SB, EK, KB, MG, and KP conducted the experiments. GA, CC, EK, SM, SB, KP, and AW analyzed and interpreted the data GA, KP, and CC drafted the manuscript. Critical revision of the manuscript for important intellectual content - all authors. GA and CC did the statistical analyses. All authors contributed to the article and approved the submitted version.

\section{FUNDING}

This work was supported by Mrs Berta Kamprad Foundation (grant no 2020-19-301), Swedish Cancer Society (grant no 20 1298), John and Augusta Persson's Foundation and governmental research funding (ST-ALF). Funding for AW was provided by the Swedish Society for Medical Research (SSMF).

\section{ACKNOWLEDGMENTS}

Parts of the results were presented at the Annual Meeting of European Radiation Research Society in Lund, Sweden, in September 2020. The content in the article is part of a PhDthesis by GA. 


\section{SUPPLEMENTARY MATERIAL}

The Supplementary Material for this article can be found online at: https://www.frontiersin.org/articles/10.3389/fonc.2021. 686142/full\#supplementary-material

\section{REFERENCES}

1. Durante M, Brauer-Krisch E, Hill M. Faster and Safer? FLASH Ultra-High Dose Rate in Radiotherapy. Br J Radiol (2017) 91:20170628. doi: 10.1259/ bjr.20170628

2. Favaudon V, Caplier L, Monceau V, Pouzoulet F, Sayarath M, Fouillade C, et al. Ultrahigh Dose-Rate FLASH Irradiation Increases the Differential Response Between Normal and Tumor Tissue in Mice. Sci Transl Med (2014) 6:245ra93. doi: 10.1126/scitranslmed.3008973

3. Montay-Gruel P, Petersson K, Jaccard M, Boivin G, Germond J-F, Petit B, et al. Irradiation in a Flash: Unique Sparing of Memory in Mice After Whole Brain Irradiation With Dose Rates Above 100 Gy/s. Radiother Oncol (2017) 124:365-9. doi: 10.1016/j.radonc.2017.05.003

4. Vozenin M-C, De Fornel P, Petersson K, Favaudon V, Jaccard M, Germond JF, et al. The Advantage of FLASH Radiotherapy Confirmed in Mini-Pig and Cat-Cancer Patients. Clin Cancer Res (2019) 25:35-42. doi: 10.1158/10780432.CCR-17-3375

5. Montay-Gruel P, Acharya MM, Gonçalves Jorge P, Petit B, Petridis IG, Fuchs $\mathrm{P}$, et al. Hypo-Fractionated FLASH-RT as an Effective Treatment Against Glioblastoma That Reduces Neurocognitive Side Effects in Mice. Clin Cancer Res (2020) 27:775-84. doi: 10.1158/1078-0432.ccr-20-0894

6. Bourhis J, Sozzi WJ, Jorge PG, Gaide O, Bailat C, Duclos F, et al. Treatment of a First Patient With FLASH-Radiotherapy. Radiother Oncol (2019) 139:1822. doi: 10.1016/j.radonc.2019.06.019

7. Wilson JD, Hammond EM, Higgins GS, Petersson K. Ultra-High Dose Rate (FLASH) Radiotherapy: Silver Bullet or Fool's Gold? Front Oncol (2020) 9:1563. doi: 10.3389/fonc.2019.01563

8. Pratx G, Kapp DS. A Computational Model of Radiolytic Oxygen Depletion During FLASH Irradiation and its Effect on the Oxygen Enhancement Ratio. Phys Med Biol (2019) 64:185005. doi: 10.1088/1361-6560/ab3769

9. Spitz DR, Buettner GR, Petronek MS, St-Aubin JJ, Flynn RT, Waldron TJ, et al. An Integrated Physico-Chemical Approach for Explaining the Differential Impact of FLASH Versus Conventional Dose Rate Irradiation on Cancer and Normal Tissue Responses. Radiother Oncol (2019) 139:23-7. doi: 10.1016/j.radonc.2019.03.028

10. Petersson K, Adrian G, Butterworth K, McMahon SJ. A Quantitative Analysis of the Role of Oxygen Tension in FLASH Radiation Therapy. Int J Radiat Oncol (2020) 107:539-47. doi: 10.1016/j.ijrobp.2020.02.634

11. Labarbe R, Hotoiu L, Barbier J, Favaudon V. A Physicochemical Model of Reaction Kinetics Supports Peroxyl Radical Recombination as the Main Determinant of the FLASH Effect. Radiother Oncol (2020) 153:1-8. doi: 10.1016/j.radonc.2020.06.001

12. Adrian G, Konradsson E, Lempart M, Bäck S, Ceberg C, Petersson K. The FLASH Effect Depends on Oxygen Concentration. Br J Radiol (2020) 93:20190702. doi: 10.1259/bjr.20190702

13. Montay-Gruel P, Acharya MM, Petersson K, Alikhani L, Yakkala C, Allen BD, et al. Long-Term Neurocognitive Benefits of FLASH Radiotherapy Driven by Reduced Reactive Oxygen Species. Proc Natl Acad Sci (2019) 116:10943-51. doi: 10.1073/pnas.1901777116

14. Buonanno M, Grilj V, Brenner DJ. Biological Effects in Normal Cells Exposed to FLASH Dose Rate Protons. Radiother Oncol (2019) 139:51-5. doi: 10.1016/ j.radonc.2019.02.009

15. Khan S, Bassenne M, Wang J, Manjappa R, Melemenidis S, Breitkreutz DY, et al. Multicellular Spheroids as In Vitro Models of Oxygen Depletion During FLASH Irradiation. Int J Radiat Oncol (2021) 110:833-44. doi: 10.1016/ j.ijrobp.2021.01.050

16. Pommarel L, Vauzour B, Mégnin-Chanet F, Bayart E, Delmas O, Goudjil F, et al. Spectral and Spatial Shaping of a Laser-Produced Ion Beam for
Supplementary Figure 1 | Cell cycle distributions determined by flow cytometry after irradiation with FLASH or conventional dose rate (CONV) for the HeLa subclone cell line $24 \mathrm{~h}$ after irradiation with $6 \mathrm{~Gy}$. The S-phase was sub-divided into S1 (early), S2 (middle) and S3 (late). Bars illustrate G1 (light grey), S-phase (grey), and G2/M (black). The figures in the bars denote the percentage of cells (with standard deviations). Data from two independent experiments.

Radiation-Biology Experiments. Phys Rev Accel Beams (2017) 20:1-10. doi: 10.1103/PhysRevAccelBeams.20.032801

17. Bayart E, Flacco A, Delmas O, Pommarel L, Levy D, Cavallone M, et al. Fast Dose Fractionation Using Ultra-Short Laser Accelerated Proton Pulses can Increase Cancer Cell Mortality, Which Relies on Functional PARP1 Protein. Sci Rep (2019) 9:10132. doi: 10.1038/s41598-019-46512-1

18. Manti L, Perozziello FM, Borghesi M, Candiano G, Chaudhary P, Cirrone GAP, et al. The Radiobiology of Laser-Driven Particle Beams: Focus on SubLethal Responses of Normal Human Cells. J Instrum (2017) C03084-C03084. doi: 10.1088/1748-0221/12/03/C03084

19. Venkatesulu BP, Sharma A, Pollard-Larkin JM, Sadagopan R, Symons J, Neri S, et al. Ultra High Dose Rate ( $35 \mathrm{~Gy} / \mathrm{sec}$ ) Radiation Does Not Spare the Normal Tissue in Cardiac and Splenic Models of Lymphopenia and Gastrointestinal Syndrome. Sci Rep (2019) 9:17180. doi: 10.1038/s41598019-53562-y

20. Colangelo NW, Azzam EI. The Importance and Clinical Implications of FLASH Ultra-High Dose-Rate Studies for Proton and Heavy Ion Radiotherapy. Radiat Res (2019) 193:1-4. doi: 10.1667/RR15537.1

21. Yamatodani T, Ekblad L, Kjellén E, Johnsson A, Mineta H, Wennerberg J. Epidermal Growth Factor Receptor Status and Persistent Activation of Akt and p44/42 MAPK Pathways Correlate With the Effect of Cetuximab in Head and Neck and Colon Cancer Cell Lines. J Cancer Res Clin Oncol (2009) 135:395-402. doi: 10.1007/s00432-008-0475-2

22. Lempart M, Blad B, Adrian G, Bäck S, Knöös T, Ceberg C, et al. Modifying a Clinical Linear Accelerator for Delivery of Ultra-High Dose Rate Irradiation. Radiother Oncol (2019) 139:40-5. doi: 10.1016/j.radonc.2019.01.031

23. McMahon SJ. The Linear Quadratic Model: Usage, Interpretation and Challenges. Phys Med Biol (2018) 64:01TR01. doi: 10.1088/1361-6560/aaf26a

24. Fouillade C, Curras-Alonso S, Giuranno L, Quelennec E, Heinrich S, BonnetBoissinot S, et al. FLASH Irradiation Spares Lung Progenitor Cells and Limits the Incidence of Radio-Induced Senescence. Clin Cancer Res (2019) 26:1497506. doi: 10.1158/1078-0432.CCR-19-1440

25. Cygler J, Klassen NV, Ross CK, Bichay TJ, Raaphorst GP. The Survival of Aerobic and Anoxic Human Glioma and Melanoma Cells After Irradiation at Ultrahigh and Clinical Dose Rates. Radiat Res (1994) 140:79-84. doi: 10.2307/ 3578571

26. Zackrisson BU, Nystrom UH, Ostbergh P. Biological Response In Vitro to Pulsed High Dose Rate Electrons From A Clinical Accelerator. Acta Oncol (Madr) (1991) 30:747-51. doi: 10.3109/02841869109092451

27. Nias AHW, Swallow AJ, Keene JP, Hodgson BW. Survival of HeLa Cells From 10 Nanosecond Pulses of Electrons. Int J Radiat Biol (1970) 17:595-8. doi: $10.1080 / 09553007014550751$

28. Berry RJ, Stedeford JBH. Reproductive Survival of Mammalian Cells After Irradiation at Ultra-High Dose-Rates: Further Observations and Their Importance for Radiotherapy. Br J Radiol (1972) 45:171-7. doi: 10.1259/ 0007-1285-45-531-171

29. Town CD. Effect of High Dose Rates on Survival of Mammalian Cells. Nature (1967) 215:847-8. doi: 10.1038/215847a0

30. Berry RJ, Hall EJ, Forster DW, Storr TH, Goodman MJ. Survival of Mammalian Cells Exposed to X Rays at Ultra-High Dose-Rates. Br J Radiol (1969) 42:102-7. doi: 10.1259/0007-1285-42-494-102

31. Epp ER, Weiss H, Djordjevic B, Santomasso A. The Radiosensitivity of Cultured Mammalian Cells Exposed to Single High Intensity Pulses of Electrons in Various Concentrations of Oxygen. Radiat Res (1972) 52:32432. doi: $10.2307 / 3573572$

32. Michaels HB, Epp ER, Ling CC, Peterson EC. Oxygen Sensitization of CHO Cells at Ultrahigh Dose Rates: Prelude to Oxygen Diffusion Studies. Radiat Res (1978) 76:510-21. doi: $10.2307 / 3574800$ 
33. Chang-Liu CM, Woloschak GE. Effect of Passage Number on Cellular Response to DNA-Damaging Agents: Cell Survival and Gene Expression. Cancer Lett (1997) 113:77-86. doi: 10.1016/s0304-3835(97)04599-0

34. Auer S, Hable V, Greubel C, Drexler GA, Schmid TE, Belka C, et al. Survival of Tumor Cells After Proton Irradiation With Ultra-High Dose Rates. Radiat Oncol (2011) 6:2-9. doi: 10.1186/1748-717X-6-139

35. Chabi S, Van TTH, Leavitt R, Poglio S, Jorge PG, Jaccard M, et al. Ultra-HighDose-Rate FLASH and Conventional-Dose-Rate Irradiation Differentially Affect Human Acute Lymphoblastic Leukemia and Normal Hematopoiesis. Int J Radiat Oncol Biol Phys (2021) 109:819-29. doi: 10.1016/ j.ijrobp.2020.10.012

36. Bourhis J, Montay-Gruel P, Gonçalves Jorge P, Bailat C, Petit B, Ollivier J, et al. Clinical Translation of FLASH Radiotherapy: Why and How? Radiother Oncol (2019) 139:11-7. doi: 10.1016/j.radonc.2019.04.008

37. Diffenderfer ES, Verginadis II, Kim MM, Shoniyozov K, Velalopoulou A, Goia D, et al. Design, Implementation, and In Vivo Validation of a Novel Proton FLASH Radiation Therapy System. Int J Radiat Oncol (2020) 106:440-8. doi: 10.1016/j.ijrobp.2019.10.049

38. Levy K, Natarajan S, Wang J, Chow S, Eggold JT, Loo PE, et al. Abdominal FLASH Irradiation Reduces Radiation - Induced Gastrointestinal Toxicity for the Treatment of Ovarian Cancer in Mice. Sci Rep (2020) 10:1-14. doi: $10.1038 /$ s41598-020-78017-7

Conflict of Interest: The authors declare that the research was conducted in the absence of any commercial or financial relationships that could be construed as a potential conflict of interest.

Publisher's Note: All claims expressed in this article are solely those of the authors and do not necessarily represent those of their affiliated organizations, or those of the publisher, the editors and the reviewers. Any product that may be evaluated in this article, or claim that may be made by its manufacturer, is not guaranteed or endorsed by the publisher.

Copyright (๔) 2021 Adrian, Konradsson, Beyer, Wittrup, Butterworth, McMahon, Ghita, Petersson and Ceberg. This is an open-access article distributed under the terms of the Creative Commons Attribution License (CC BY). The use, distribution or reproduction in other forums is permitted, provided the original author(s) and the copyright owner(s) are credited and that the original publication in this journal is cited, in accordance with accepted academic practice. No use, distribution or reproduction is permitted which does not comply with these terms. 